\title{
QUANTITATIVE ANALYSIS IN HEURISTIC EVALUATION EXPERIMENTS OF E-COMMERCE WEBSITES
}

\author{
Xiaosong Li, Ye Liu, Zizhou Fan and Will Li \\ Computer Science Practice Pathway, \\ Unitec Institute of Technology, Auckland, New Zealand
}

\begin{abstract}
This paper reports a pilot study on developing an instrument to predict the quality of ecommerce websites. The $8 C$ model was adopted as the reference model of the heuristic evaluation. Each dimension of the $8 C$ was mapped into a set of quantitative website elements, the websites were scraped to get the quantitative website elements, and the score of the dimension was calculated. A software was developed in PHP for the experiments. In the training process, 10 experiments were conducted and quantitative analysis was regressively conducted between the experiments. The conversion rate was used to verify the heuristic evaluation of an e-commerce website. The results showed that the mapping revisions between the experiments improved the performance of the evaluation instrument, therefore the experiment process and the quantitative mapping revision guideline proposed was on the right track. The experiment results and the future work have been discussed.
\end{abstract}

\section{KEYWORDS}

E-commerce Website, Heuristic Evaluation, Regression Experiments, 8C framework, Quantitative Analysis

\section{INTRODUCTION}

E-commerce websites have increased greatly in the new era; they face many competitors. Research revealed that efforts put into usability design and modification improved the performance of usability on websites greatly [1]. To help website developers and other stakeholders understand how to develop e-commerce websites properly and maximize profit, many evaluation methods have been developed $[1,2]$. One approach is called user based testing [1], which takes into account subjective perception, both in terms of website content and design. This perception varies with the expertise, the cognitive skills and the end goal of each user [1]. If an automatic approach is used to evaluate website content and design from the user's perspective, that should standardize the evaluation process and make the evaluation consistent and objective.

7C framework was introduced to evaluate the quality of e-commerce website content and user interface design [3], which is considered as a useful reference model for developers, analysts, managers, and executives, when designing and/or evaluating the interface channels between the customer and the web based application. However, it is insufficient to completely address the new generation of web applications [4]. Collaboration and user-generated content are important features in the new generation websites. The $7 \mathrm{C}$ framework was extended into the $8 \mathrm{C}$ framework by adding collaboration as the 8th element in the model and the meaning of each of the eight

David C. Wyld et al. (Eds) : CNDC, DPPR, AIAA, WiMNET, ACITY - 2017

pp. 71- 80, 2017. (C) CS \& IT-CSCP 2017

DOI : $10.5121 /$ csit.2017.71607 
design elements was updated as well, so that they are effective in representing the interface design elements of new generation websites [4].

Usually when a website is evaluated against the 7C framework, subjective perception is used. For example, in [5], a checklist consisting of 63 checkpoints was developed based on research literature and expert opinions to evaluate a group of 4 and 5-star luxury hotel websites against the 7C framework. This approach again could be inconsistent and subjective. An automatic approach could improve this.

The heuristic evaluation method is a technique for evaluating the usability, with the inspection being carried out mainly by evaluators from principles established by the discipline [6]. In most applications the results tend to be qualitative, however, these qualitative results do not allow us to determine how usable it is or how it becomes an interactive system. Hence, the need for quantitative results may also be very necessary in order to determine the effort that would be needed to get a sufficiently usable system [6].

The accurate prediction of a numerical target variable is an important task in machine learning. Quantitative heuristic analysis has been used in machine learning to predict various values in the data mining and inductive rule learning communities, where a strong focus lies on the comprehensibility of the learned models [7]. In [7], a heuristic rule learning algorithm that learns regression models is used where a region around the target value predicted by the rule is dynamically defined. In [8], a unified measure of web usability was used based on a multiple regression model, and then the estimated index is used to measure its impact on community bank performance. Results showed that banks with higher usability score perform significantly better than those with lower score.

Conversion rate (CR) is the percentage of users who take a desired action. The typical example of conversion rate is the percentage of website visitors who buy something on the site, For the purpose of managing user interface design and tracking the effectiveness of user experience efforts, the conversion rate is usually very important [9]. The conversion rate measures what happens once people are at your website, which is greatly influenced by the design and is a key parameter to track for assessing whether a user experience strategy is working. Lower conversion rates? You must be doing something wrong with the design. Higher conversion rates? You can praise your designers [9]. This suggests that there is a proportional relationship between the conversion rate of an e-commerce website and its user interface design. It is reasonable to use the conversion rate to measure the quality of the user interface of an e-commerce website.

This paper presents a pilot study on developing an instrument to predict the quality of ecommerce websites. The objective of the resulting instrument is to provide a meaningful estimation on the quality of a given e-commerce website. The $8 \mathrm{C}$ model was adopted as the reference model of the heuristic evaluation. Each dimension of the $8 \mathrm{C}$ model was mapped into a formula consisting of a set of quantitative website elements, the websites were scraped to get the quantitative website elements, and the score of the dimension was calculated based on the formula. Another formula was defined to calculate the total score for the website based on the scores from each dimension.

A software was developed in PHP for both training and testing experiments. An experimental process and its quantitative mapping revision guideline were proposed and used. In the training process, 10 experiments were conducted and quantitative analysis was regressively conducted between the experiments. The conversion rate was used in this study to test and verify the heuristic evaluation of an e-commerce website. 100 websites from five different categories were selected as the training data. 7 websites ordered by the conversion rate were used as testing data 
to test the results at the end of each experiment in the training process and 15 websites ordered by the CR were used as the testing data.

In the rest of this paper, the design of the experiment is described first, then the experiments and the results are presented and discussed, after that a summary and future work are given lastly.

\section{THE EXPERIMENT ENVIRONMENT AND DESIGN}

This study considered the seven dimensions defined in the $7 \mathrm{C}$ framework and the additional dimension "collaboration" introduced in the 8C framework. For the web 2.0 features, only those features easy to be obtained via web scraping were considered such as website forum, blog and Ajax. Table 1 presents the key meaning of each dimension in 8C [4].

Table 1. The key meaning of each dimension in $8 \mathrm{C}$.

\begin{tabular}{|l|l|}
\hline Dimensions & Meanings \\
\hline 1: Context & How the site is organized, and how the content is presented to the users? \\
\hline 2: Content & What are offered by the site? \\
\hline 3: Community & Non-interactive communication; Interactive communication. \\
\hline 4: Customization & Refers to the site's ability to tailor itself (tailoring) or to be tailored. \\
\hline 5: Communication & Site-to-user communications. \\
\hline 6: Connection & Refers to the extent of formal linkage from one site to others. \\
\hline 7: Commerce & Deals with the interface that supports the various aspects of e-commerce. \\
\hline 8: Collaboration & Generally in the form of feedback forms, forums, and bulletin boards. \\
\hline
\end{tabular}

Quantitative usability estimation is typically associated with the calculation of metrics that assess dimensions of software quality [6]. Measuring the user experience offers so much more than just simple observation. Metrics add structure to the design and evaluation process, give insight into the findings and provide information to the decision makers [10].

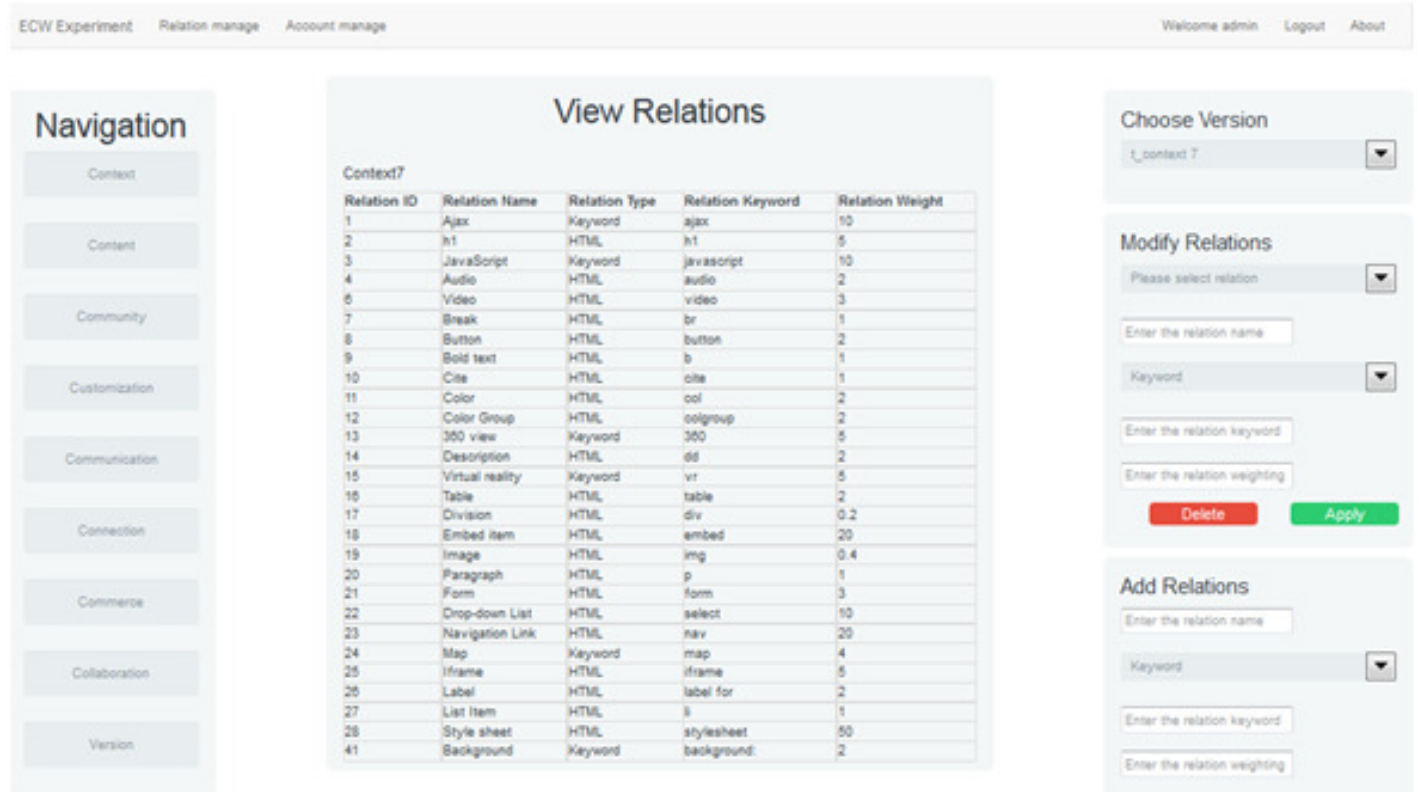

Figure 1. The mapping management UI and the relations in a mapping 
A software written in PHP was developed for both training and testing experiments. Figure 1 shows the software mapping management user interface with the mapping relations between the Context dimension and the selected HTML tags/keywords in Experiment 7. For an e-commerce website to be experimented, only the home page was considered in this study.

Two major approaches were used to identify the website quantitative elements and calculate the metrics for each dimension: finding keywords and scraping HTML tags, where a keyword could be an important text or a JavaScript/CSS keyword. Each keyword or HTML tag is associated with a numeric weight, which determines the importance of the relation, higher weight means more important. The mapping relations between each dimension and the selected keyword or HTML tag are defined before each experiment, which can be adjusted in the subsequent experiments based on the experiment results.

Let $N R$ be the total number of the relations in a mapping between a dimension and the selected HTML tags/keywords; $R S i$ be the score of relation $i$; Wi be the associated weight of relation $i$; if the relation $i$ is a keyword, $R S i$ will be $W i$; if relation $i$ is an HTML tag, $R S i$ will be calculated by the following formula:

$$
R S i=\frac{S T a g N i}{\operatorname{TTagNi}} * \text { Scalar } * W i
$$

Where STagNi is the number of the occurrence of the selected HTML tag for relation $i$; TTagNi is the total number of HTML tag on the selected page; Scalar is set as 100 to make the score a meaningful magnitude. The total score $T S$ is the sum of the scores for all 8 dimensions in $8 \mathrm{C}$ framework.

$$
T S=\sum_{i=1}^{8}(R S i)
$$

An experimental process and its revision guideline were proposed and used. Initially, in Experiment 1, only the keywords/HTML tags that can intuitively reflect the meaning of a dimension as defined in the $8 \mathrm{C}$ framework were selected as the relations for the mapping of that dimension heuristically. The weights for the relations also were selected in the similar way heuristically.

Then the scores for all the training websites were calculated respectively according to formula (2). The training websites were ordered based on their CRs (CR) first, and then the training websites were ordered again based on their scores. If the score order is different from the CR order, the mappings for all the 8 dimensions were reviewed and revised in the following three aspects:

1. Check if any relation score is dominating the dimension score based on the overall performance of the training websites, if yes, adjust the weight of that relation to make the relation score of a meaningful magnitude.

2. Check if the score of any dimension is dominating the total score based on the overall performance of the training websites, if yes, scale all the scores in that dimension to make the dimension score of a meaningful magnitude.

3. Recheck all the mappings against the $8 \mathrm{C}$ model and make adjustment accordingly. This may involve adding or deleting relations. 
The above would result in the new mappings for the next experiment. This process went through regressively for 10 experiments. As an example, Table 2 shows the mappings for Collaboration dimension in Experiment 1, Experiment 6 and Experiment 8.

Table 2. The mapping for Collaboration in three experiments.

\begin{tabular}{|l|l|l|l|l|l|}
\hline \multicolumn{2}{|l|}{ Experiment 1 } & Experiment 6 & Experiment 8 \\
\hline $\begin{array}{l}\text { Relation } \\
\text { Name }\end{array}$ & $\begin{array}{l}\text { Relation } \\
\text { Weight }\end{array}$ & Relation Name & $\begin{array}{l}\text { Relation } \\
\text { Weight }\end{array}$ & Relation Name & $\begin{array}{l}\text { Relation } \\
\text { Weight }\end{array}$ \\
\hline Forums & 3 & Forums & 3 & Forums & 3 \\
\hline Bulletin boards & 3 & Bulletin boards & 3 & Bulletin boards & 3 \\
\hline FAQ & 3 & FAQ & 3 & FAQ & 3 \\
\hline & & Feedback & 5 & Feedback & 5 \\
\hline & & & & Review & 5 \\
\hline & & & & Suggestion & 5 \\
\hline & & & & Comment & 5 \\
\hline
\end{tabular}

\section{THE EXPERIMENT ENVIRONMENT AND DESIGN}

100 websites from five different categories (Electronics, Publishing \& entertainment, Home and garden, Books, Industrial equipment), 20 from each category were selected as the training data. The five categories were selected from [11], where the CRs for 25 retail categories were listed. Electronics and Publishing \& entertainment were associated with high level CR; Home \& garden and Books were associated with middle level CR; and Industrial equipment were associated with low level CR

Table 3. The categories of training data.

\begin{tabular}{|l|l|}
\hline Categories & Conversion Rates \\
\hline Electronics & Around 23\% \\
\hline Publishing \& entertainment & Around 20\% \\
\hline Home \& garden & Around 14\% \\
\hline Books & Around 13\% \\
\hline Industrial equipment & Around 7\% \\
\hline
\end{tabular}

The top 10 e-commerce websites based on CR for 2010 were listed in [12], only 7 of them were valid for the experiments, and they all were used to test the results at the end of each experiment for all the 10 experiments. Table 4 shows the 7 testing websites.

Table 4. The testing data.

\begin{tabular}{|l|l|}
\hline Website Names & Conversion Rates \\
\hline Woman Within & $25.3 \%$ \\
\hline Blair & $20.4 \%$ \\
\hline 1800petmeds & $17.7 \%$ \\
\hline qvc & $16 \%$ \\
\hline ProFlowers & $15.8 \%$ \\
\hline Oriental Trading Company & $14.9 \%$ \\
\hline Roamans & $14.4 \%$ \\
\hline
\end{tabular}

After each experiment, the training websites were ordered again based on their scores. If the score order is different from the $\mathrm{CR}$ order, the mappings for all the 8 dimensions in $8 \mathrm{C}$ model were reviewed and then revised if needed, this resulted in the new mapping for the next experiment. Figure 2 shows the absolute score differences between the expected order and the actual order. [9] 
suggests that there is a proportional relationship between the CR of an e-commerce website and its user interface design. It is reasonable to assume that the less the difference, the more accurate the evaluation. The differences in each experiment for all the 7 training websites were averaged and Figure 3 shows the average for all the experiments except Experiment 9. As the scores of Experiment 10 were obtained by scaling the scores in Experiment 9 by 10\%. It was observed that the trends of the curve going down along the experiments. This suggested that the mapping revisions between the experiments improved the performance of the evaluation instrument and it is positive.

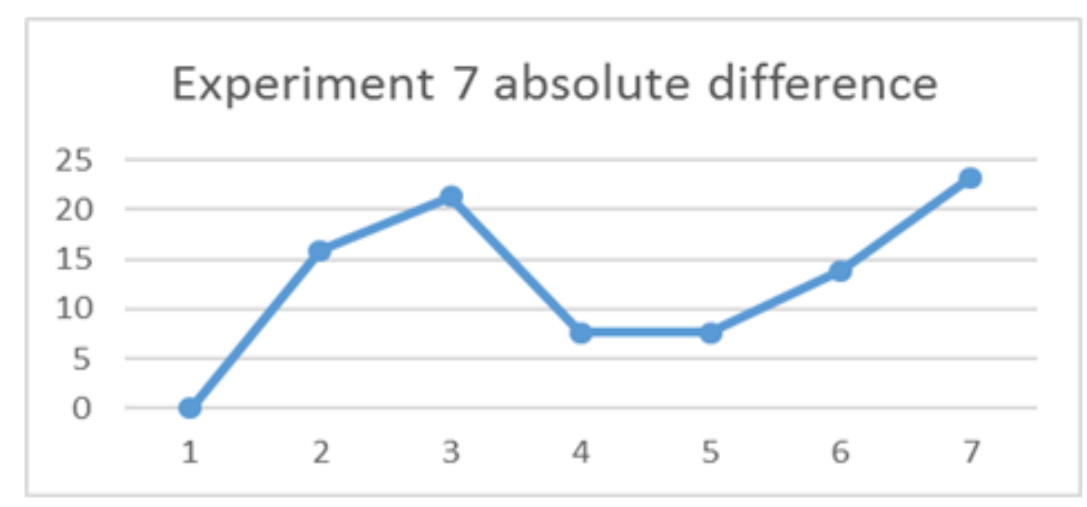

Figure 2. The absolute score differences between the expected order and the actual order for one experiment

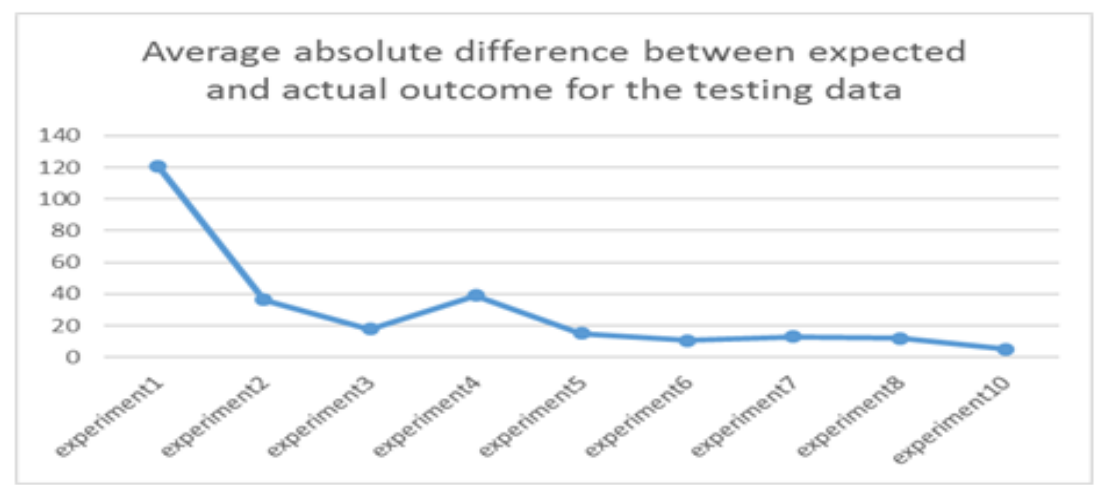

Figure 3. The average absolute difference between expected and actual outcomes for nine experiments

Table 5. The dimension contribution analysis of Experiment $8 \& 10$.

\begin{tabular}{|l|l|l|l|l|l|l|}
\hline & \multicolumn{4}{|l|}{ Experiment 8 } & Experiment 10 \\
\hline $\begin{array}{l}\text { Attribute } \\
\text { Category }\end{array}$ & $\begin{array}{l}\text { Attribute } \\
\text { Number }\end{array}$ & $\begin{array}{l}\text { Contribution } \\
\text { to the total } \\
\text { score }\end{array}$ & $\begin{array}{l}\text { Standard } \\
\text { deviation }\end{array}$ & $\begin{array}{l}\text { Attribute } \\
\text { Number }\end{array}$ & $\begin{array}{l}\text { Contribution } \\
\text { to the total } \\
\text { score }\end{array}$ & $\begin{array}{l}\text { Standard } \\
\text { deviation }\end{array}$ \\
\hline Context & 28 & $40.94 \%$ & 15.43 & 28 & $14.71 \%$ & 1.55 \\
\hline Content & 11 & $5.93 \%$ & 7.08 & 11 & $8.21 \%$ & 2.62 \\
\hline Community & 18 & $9.04 \%$ & 7.77 & 18 & $12.60 \%$ & 3.13 \\
\hline Customization & 5 & $7.85 \%$ & 6.00 & 5 & $11.22 \%$ & 2.43 \\
\hline Communication & 13 & $12.47 \%$ & 6.55 & 13 & $17.79 \%$ & 2.65 \\
\hline Connection & 2 & $5.15 \%$ & 4.81 & 2 & $7.44 \%$ & 1.97 \\
\hline Commerce & 10 & $14.68 \%$ & 8.30 & 10 & $15.80 \%$ & 2.55 \\
\hline Collaboration & 7 & $3.93 \%$ & 3.94 & 7 & $12.22 \%$ & 3.48 \\
\hline
\end{tabular}


In Experiment 8, it was observed that some of the dimensions' scores dominated the total score of the website. Table 5 shows the dimension contribution analysis of Experiment $8 \& 10$, where the number of attributes number is the number of relations in the mapping for each dimension (Attribute Category) of the $8 \mathrm{C}$; contribution to the total score is the sum of the scores in a dimension for all the training websites divided by the total score of all the training websites in an experiment. Context made much more contribution (40.94\%) than the others did. On the other hand, some were too small to influence the total score, such as Content $(5.93 \%)$ Connection (5.93\%) and Collaboration (5.93\%). The standard deviation can provide some ideas on whether the attributes in a dimension is informative. For example, standard deviation for Collaboration was the smallest one in Experiment 8, however, there were 7 attributes in this dimension. This suggested that the meaning of the attributes might be overlapping. So standard deviation for each dimension over all the training websites should be considered in the review process after each experiment in the future study.

In this study, scaling the scores for the dimensions were attempted to balance the influences of all the dimensions. For a website, let TS be its total score, and let score codes and scale parameter codes be defined in Table 6 .

Table 6. Codes used in the scale formula.

\begin{tabular}{|l|l|l|l|}
\hline $\begin{array}{l}\text { Score } \\
\text { Code }\end{array}$ & Meaning of the code & $\begin{array}{l}\text { Scale } \\
\text { Parameter }\end{array}$ & Scale Number \\
\hline SC1 & Score of Context & P1 & 1 \\
\hline SC2 & Score of Content & P2 & 4 \\
\hline SC3 & Score of Community & P3 & 4 \\
\hline SC4 & Score of Customization & P4 & 4 \\
\hline SC5 & Score of Communication & P5 & 4 \\
\hline SC6 & Score of Connection & P6 & 4 \\
\hline SC7 & Score of Commerce & P7 & 3 \\
\hline SC8 & Score of Collaboration & P8 & 9 \\
\hline
\end{tabular}

Formula (3) was used to calculate TS in Experiment 9, the resulting scores were much larger than the other experiments, so the results were divided by 10 for further scaling, which were recorded as Experiment 10.

$$
T S=\sum_{i=1}^{8}(S C i * P i)
$$

The right column of Table 5 shows the contribution of each dimension after the scaling in Experiment 10. This time the contributions of the dimensions are much balanced.

The verifying data was obtained from [13], which listed top 15 e-commerce websites based on CR for 2014. All of them were valid for the experiments and were used to check the mappings used in all the experiments except Experiment 9 as Experiment 10 can represent Experiment 9. Table 7 shows the order of the 15 verifying websites. Figure 4 shows the average absolute difference between expected and actual outcomes for the 15 verifying websites. It was observed that the trend of the curve was going down along the experiments, which was consistent with the testing results of Figure 3. This suggested that the experiments were on the right track and the results were positive. The resulting instrument from Experiment 10 could be used to evaluate a given e-commence website and provide meaningful estimation on the quality of the website. 
Table 7. The 2014 data.

\begin{tabular}{|l|l|}
\hline Website Names & Conversion Rates \\
\hline Play.Google & $30.00 \%$ \\
\hline MovieMars & $22.95 \%$ \\
\hline DollarShaveClub & $20.00 \%$ \\
\hline 1800Contacts & $18.40 \%$ \\
\hline 1800Flowers & $16.90 \%$ \\
\hline Coastal & $14.50 \%$ \\
\hline Keurig & $13.00 \%$ \\
\hline FTD & $11.70 \%$ \\
\hline ProFlowers & $11.70 \%$ \\
\hline PureFormulas & $10.74 \%$ \\
\hline FreshDirect & $10.50 \%$ \\
\hline TheGreatCourses & $10.04 \%$ \\
\hline 1800PetMeds & $10.00 \%$ \\
\hline AmeriMark & $10.00 \%$ \\
\hline OvernightPrints & $9.95 \%$ \\
\hline
\end{tabular}

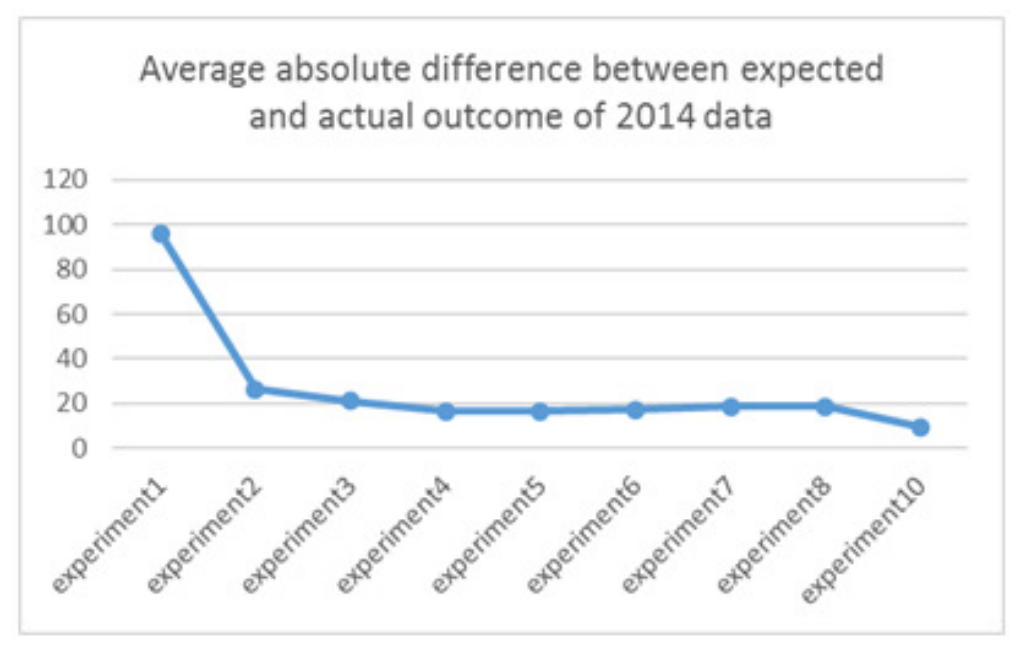

Figure 4. The average absolute difference between expected and actual outcomes for 2014 data.

Table 8. The average score of each category in each experiment.

\begin{tabular}{|l|l|l|l|l|l|}
\hline No & Electronics & Entertainment & Home & Books & Industrial \\
\hline 1 & 165.27 & 142.92 & 157.36 & 152.41 & 148.19 \\
\hline 2 & 144.16 & 124.11 & 124.71 & 129.84 & 124.40 \\
\hline 3 & 108.77 & 83.19 & 85.73 & 93.20 & 88.15 \\
\hline 4 & 98.73 & 85.15 & 90.07 & 91.09 & 94.00 \\
\hline 5 & 90.08 & 77.28 & 82.28 & 87.61 & 83.50 \\
\hline 6 & 91.27 & 81.50 & 84.73 & 86.80 & 89.00 \\
\hline 7 & 163.41 & 141.10 & 145.65 & 136.69 & 148.75 \\
\hline 8 & 159.83 & 138.54 & 142.54 & 134.37 & 145.52 \\
\hline 9 & 467.53 & 395.98 & 380.85 & 358.28 & 403.04 \\
\hline 10 & 46.77 & 39.89 & 40.21 & 36.00 & 40.34 \\
\hline
\end{tabular}

Table 8 shows the average score of each category in each experiment. According to Table 3, websites in Electronics category should have the highest scores; websites in Industrial 
Equipment category should have the lowest scores; and Books are in the middle. In Table 8, the Electronics websites always have the highest score in all the experiments, Books websites are in the middle sometimes, particularly in Experiment 10. These are consistent between the two tables (Table 3 and Table 8). However, Industrial Equipment websites usually do not have the lowest scores. This suggests that the website design and usability could have an impact on an ecommerce website's CR, however, there are other factors as well, such as the product nature, those relevant factors should be taken into consideration as well in an e-commerce website evaluation. In addition, the experiment results are dynamic; they are impacted by the network environment. The quantitative mappings might not be available temporarily for those popular websites due to heavy network traffic sometimes, and those popular websites are likely the websites with high scores. Industrial Equipment websites are not as popular as book websites or entertainment websites, so they are less impacted by network traffic; on the other hand, book websites or entertainment websites might get lower scores than their real scores due to network traffic, this issue should be addressed in the future experiment.

\section{SUMMARY AND FUTURE WORK}

This paper presented a pilot study on developing an instrument to predict the quality of ecommerce websites. The objective is to provide a meaningful estimation of a given e-commerce website. The $8 \mathrm{C}$ model was adopted as the reference model of the heuristic evaluation. Each dimension of the $8 \mathrm{C}$ was mapped into quantitative elements by means of web scraping. A software was developed in PHP for both training and testing experiments. 10 experiments were conducted and quantitative analysis was regressively conducted between the experiments. The conversion rate was used to test and verify the heuristic evaluation. It was observed that the trends of the curve for the differences between the expected and actual outcomes was going down along the experiments for both of the testing data and verifying data. This suggested that the mapping revisions between the experiments improved the performance of the evaluation instrument, therefore the experiment process and the revision guideline proposed in Section 2 was on the right track.

However, there are limitations in this study. The experiments only had been done on the home page of each website, although home page is very important for a website and it can provide rich information about the website, it is not sufficient for an e-commerce website, in some cases, the shopping cart or product list are not on the home page. Due to technique incapacity, not all the website features can be mapped into quantitative elements. The experiment results could be impacted by the network environment although that impact is not significant.

The above should be considered in the future work. In addition to that, the mapping revision process could be more robotic by improving the revision guideline (algorithm), for example, the standard deviation for each dimension over all the training websites could be considered in the review process after each experiment in the future work. The evaluation framework should not be limited to the $8 \mathrm{C}$ model; it could be extended to include other factors. [13] proposed a number of ways to improve the $\mathrm{CR}$ of an e-commerce website, which should be considered in the future study.

\section{REFERENCES}

[1] Li, Fangyu, \& Yefei Li, (2011) "Usability evaluation of e-commerce on B2C websites in China.", Procedia Engineering 15 pp5299-5304.

[2] Bezes, Christophe (2009) "E-commerce website evaluation: a critical review." 
[3] Rayport, Jeffrey F., \& Bernard J. Jaworski (2002) Introduction to e-commerce. McGraw-Hill/Irwin marketspaceU.

[4] Yang, T. Andrew, Dan J. Kim, Vishal Dhalwani, \& Tri K. Vu, (2008) "The 8C framework as a reference model for collaborative value Webs in the context of Web 2.0." In Hawaii International Conference on System Sciences, Proceedings of the 41st Annual, pp319-319. IEEE.

[5] Hamidizadeh, Mohammad R., Mohammad E. Fadaeinejad, \& Fayegh Mojarrad, (2011) "Design of internet marketing based on 7Cs model." In 2011 International Conference on Social Science and Humanity.

[6] González, Marta, Llúcia Masip, Antoni Granollers, \& Marta Oliva, (2009) "Quantitative analysis in a heuristic evaluation experiment." Advances in Engineering Software 40, no. 12. pp1271-1278.

[7] Janssen, Frederik \& Johannes Fürnkranz, (2011) "Heuristic rule-based regression via dynamic reduction to classification." In IJCAI Proceedings-International Joint Conference on Artificial Intelligence, vol. 22, no. 1, pp1330.

[8] Acharya, Ram N., Albert Kagan, Srinivasa Rao Lingam \& Kevin Gray, (2011) "Impact Of Website Usability On Performance: A Heuristic Evaluation Of Community Bank Homepage Implementation." Journal of Business \& Economics Research (JBER) 6, no. 6.

[9] Nielsen, Jakob, (2013) Conversion Rates. https://www.nngroup.com/articles/conversion-rates/, last accessed 2017/06/28.

[10] Tullis, Thomas \& Albert, William. Measuring the user experience. Morgan Kaufmann; 2008.

[11] Burstein, Daniel. (2015) Ecommerce Research Chart: Industry benchmark conversion rates for 25 retail categories. http://www.marketingsherpa.com/article/chart/conversion-rates-retail-categories/, last accessed 2016/07/29.

[12] Chopra, Paras. (2010) Top 10 eCommerce Websites (by Conversion Rate). https://vwo.com/blog/topecommerce-websites-conversion-rate/, last accessed 2016/07/29.

[13] Saleh, Khalid. (2017) The Average Website Conversion Rate by Industry. https://www.invespcro.com/blog/the-average-website-conversion-rate-by-industry/, last accessed 2017/05/29.

\section{AUTHORS}

Dr. Xiaosong Li obtained her PhD (1999) in Computer Science from University of Auckland in New Zealand. Her research interests include Graphical User Interface, E-Commerce Websites, Machine Learning and etc. She joined Unitec in 2002 where she is a Senior Academic Staff Member.

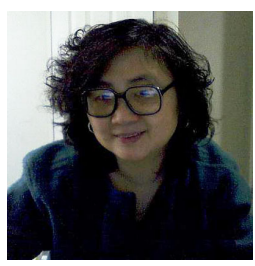

